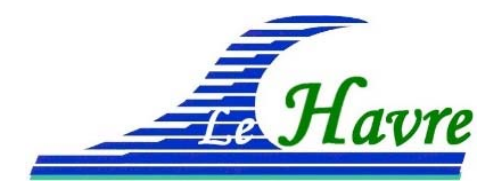

XVI ${ }^{\text {èmes }}$ Journées Nationales Génie Côtier - Génie Civil
Le Havre, 2020

DOI:10.5150/jngcgc.2020.078 (C) Editions Paralia CFL

disponible en ligne - http://www.paralia.fr - available online

\title{
Influence du set-up de houle dans le phénomène combiné de franchissement/submersion
}

\section{Syrine BEN JEBRIA ${ }^{1}$, Adrien POUPARDIN ${ }^{1}$, Philippe SERGENT ${ }^{2}$}

1. Institut de Recherche en Constructibilité, Ecole Spéciale des Travaux Publics, Université Paris-Est. 6-8 avenue Blaise-Pascal Cité Descartes - Champs-sur-Marne 77455 Marne-la-Vallée, France.

apoupardin@estp-paris.eu

\section{CEREMA EMF}

134 rue de Beauvais, CS 60039, 60280 Margny lès Compiègne Cedex, France. philippe.sergent@cerema.fr

\section{Résumé :}

Les résultats issus des expériences ont montré une bonne reproduction du set-up dans le canal, une bonne reproduction des débits de franchissement seul et de submersion seule, ainsi qu'une bonne reproduction du débit de franchissement/submersion combinés pour la gamme de $R c / H[-1.5 ;-0.5]$.

Nous avons en outre confirmé l'importance de la prise en compte de l'effet des vagues lors d'un épisode de surverse lorsque la revanche est faible. La distance entre le déferlement et la crête de digue joue également un rôle important sur la possibilité de développement du set-up en amont de la digue.

\section{Mots-clés :}

Franchissement, Submersion, Set-up, Digues, Tempêtes, Surcotes, Houle.

\section{Introduction}

Le changement climatique et en particulier la remontée du niveau des océans dont il est la cause expose le littoral et la population qu'il abrite à des risques accrus de submersion et de dommages dus au franchissement des vagues au-dessus des rivages ou des structures de protection des côtes dont le dimensionnement n'avait pas anticipé une telle évolution. De nombreux auteurs ont quantifié les débits pouvant franchir une structure en fonction des paramètres structurels de l'ouvrage et des caractéristiques de la houle le sollicitant. VAN DER MEER \& JANSSEN (1995) exprime le débit franchissant $q_{w}$ par :

$\frac{q_{w}}{\sqrt{g H_{S}^{3}}}=a \exp \left(-b \frac{R_{c}}{H_{S}}\right)$

avec $H_{S}$ la hauteur de houle significative, $R_{c}$ la revanche de la digue, $a$ et $b$ des paramètres à fixer qui peuvent dépendre du déferlement et des caractéristiques de la digue. 


\section{Thème 7 - Risques côtiers}

De même, les ingénieurs disposent depuis longtemps de formules analytiques estimant les débits passant au-dessus des déversoirs. Dans le cas de HENDERSON (1966), le débit de surverse $q_{s}$ est donné par :

$q_{s}=0.5443 \sqrt{g} h_{0}{ }^{3 / 2}$

avec $h_{0}$ la hauteur d'eau au-dessus du niveau de la digue.

Ce n'est que récemment que des chercheurs ont abordé le problème de la combinaison d'une submersion et d'un franchissement des vagues. REEVES et al. (2008) ont exprimés le débit de franchissement/submersion combiné $q_{w s}$ par :

$\frac{q_{w s}}{\sqrt{g H_{S}^{3}}}=c \exp \left(-d \frac{R_{c}}{H_{S}}\right)$

avec $c$ et $d$ des paramètres à fixer qui peuvent dépendre du déferlement et des caractéristiques de la digue.

Dans ce dernier cas, il faut être précautionneux sur le choix des formules à considérer pour déterminer le débit d'eau réel au-dessus d'une digue. Dans certaines configurations, les débits de franchissement peuvent atteindre trois fois les valeurs de débit de submersion et changent considérablement les scenarii d'inondation (HUGHES \& NADAL, 2009).

Le déferlement précoce des vagues impacte également ce débit puisqu'il est intégré à la formule de franchissement submersion (REEVES et al., 2008).

Dans le cadre de ce travail, nous avons souhaité reproduire le phénomène de franchissement/submersion en laboratoire pour comparer les résultats aux lois de la littérature précédemment cités (VAN DER MEER \& JANSSEN, 1995 ; HENDERSON, 1966 ; REEVES et al., 2008). Dans un premier temps nous avons reproduit le phénomène de set-up sans ouvrage. Puis, nous avons intégré un obstacle et mesuré les débits de franchissement et de submersion. Enfin une analyse des résultats nous a permis d'appréhender les différents phénomènes mis en œuvre générant les débits totaux de franchissement et de surverse.

\section{Protocole expérimental}

Afin de réaliser cette étude, nous avons utilisé un canal hydraulique de $9 \mathrm{~m}$ de longueur utile, de $0.41 \mathrm{~m}$ de largeur et de $0.55 \mathrm{~m}$ de hauteur, inclinable entre -0.5 et $+2.5 \%$. Le canal est équipé d'un batteur à houle monochromatique permettant de générer des houles de fréquence et d'amplitude ajustables.

Afin de mesurer la houle, nous utilisons des capteurs ultrasons Baumer qui nous donnent la hauteur d'eau locale à une fréquence d'échantillonnage de $100 \mathrm{~Hz}$.

\subsection{Mesure du set-up}

Dans un premier temps, nous avons souhaité vérifier la capacité de notre canal à reproduire le phénomène de set-up en faisant varier la pente $\alpha$ du canal et la hauteur des vagues $H$. 


\section{XVİ̀mes Journées Nationales Génie Côtier - Génie Civil \\ Le Havre 2020}

Pour réaliser le profil de hauteur d'eau moyenne dans le canal nous avons réalisé 15 acquisitions avec 6 capteurs ultrasons que nous avons déplacés entre chaque acquisition de manière à couvrir toute la longueur du canal (figure 1).

Ces mesures de set-up ont ensuite été comparées aux formules de la littérature (GODA, 1970 ; WEGGEL, 1972 ; RATTANAPITIKON \& TOMOYA, 2000) permettant de déterminer l'évolution de l'amplitude des vagues en fonction de la pente du fond. A partir de là, on retrouve la surélévation moyenne de la surface libre en utilisant l'équation de quantité de mouvement moyennée en faisant l'hypothèse des ondes longues :

$g \frac{\partial \bar{\eta}}{\partial x}=-\frac{1}{\rho(\bar{\eta}+d)} \frac{\partial S x x}{\partial x}$

avec $S x x=\frac{1}{8} \rho g H^{2}\left[\frac{1}{2}+\frac{2 k d}{\sinh (2 k d)}\right]$ la contrainte de radiation, $\bar{\eta}$ valeur moyenne de $\eta$ qui est la surélévation de la surface libre, $d$ la profondeur d'eau locale et $k=\frac{2 \pi}{\lambda}$ le nombre d'onde.

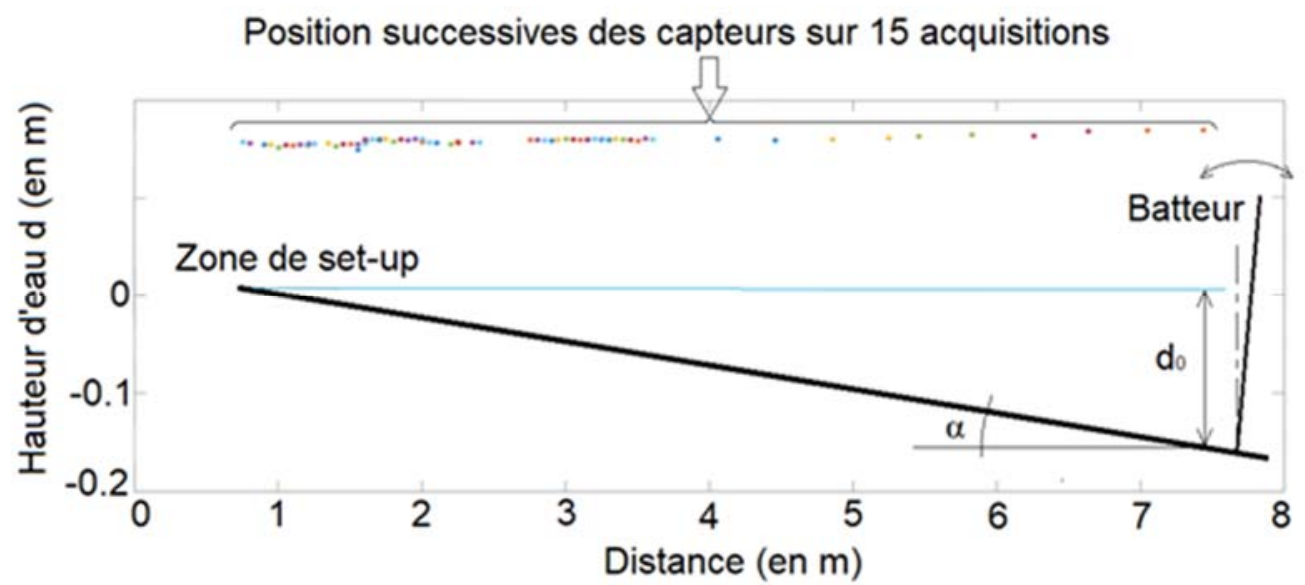

Figure 1. Points de mesure dans la direction longitudinale, (6 capteurs sur 15 acquisitions).

\subsection{Franchissement/submersion de digue}

Pour l'étude de la surverse, du franchissement et des deux combinés, nous avons considéré une digue de $20 \mathrm{~cm}$ de hauteur avec une pente de talus de $33^{\circ}$. La pente du fond $\alpha$ a été fixée à $1.5 \%$. Afin de permettre une mesure précise du volume d'eau passant pardessus la digue, nous avons dimensionné un réservoir d'une section horizontale $L \times l$, de $84 \times 39 \mathrm{~cm}^{2}$.

Dans le cas de la surverse, une pompe, de rayon $R=6.5 \mathrm{~cm}$ et de débit $Q=8.6 \mathrm{~m}^{3} / \mathrm{h}$, immergée dans ce réservoir, renvoyait l'eau dans le canal (en amont de la digue).

Des capteurs ultrasons étaient positionnés en amont de la digue, pour mesurer la houle, et au-dessus du réservoir pour mesurer les variations de niveau induits par des débits franchissants notamment. 


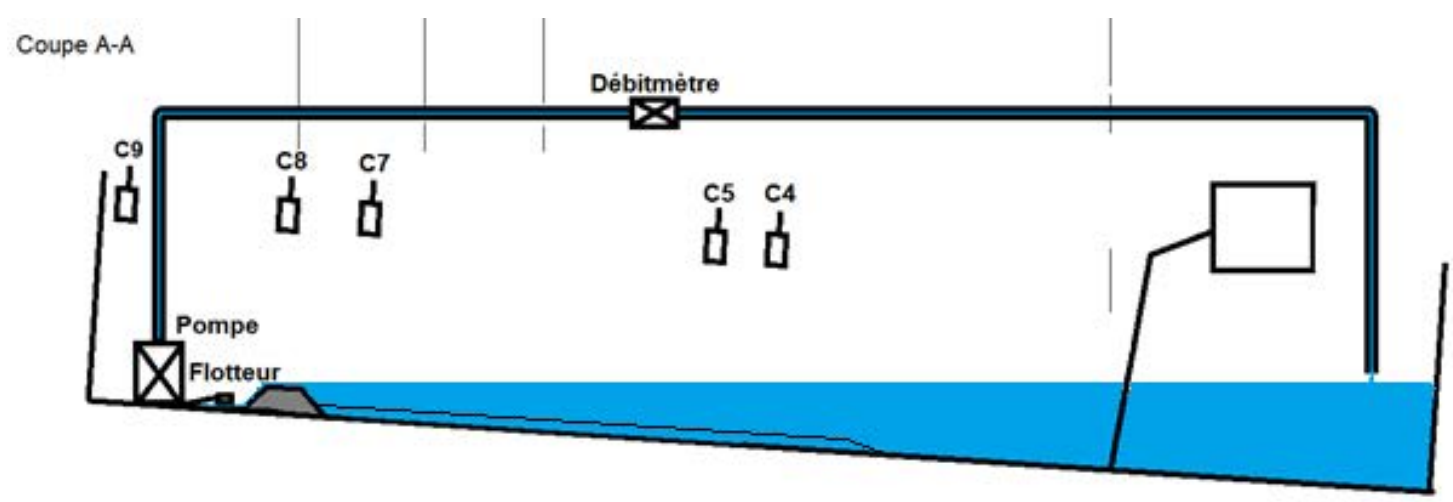

(a)

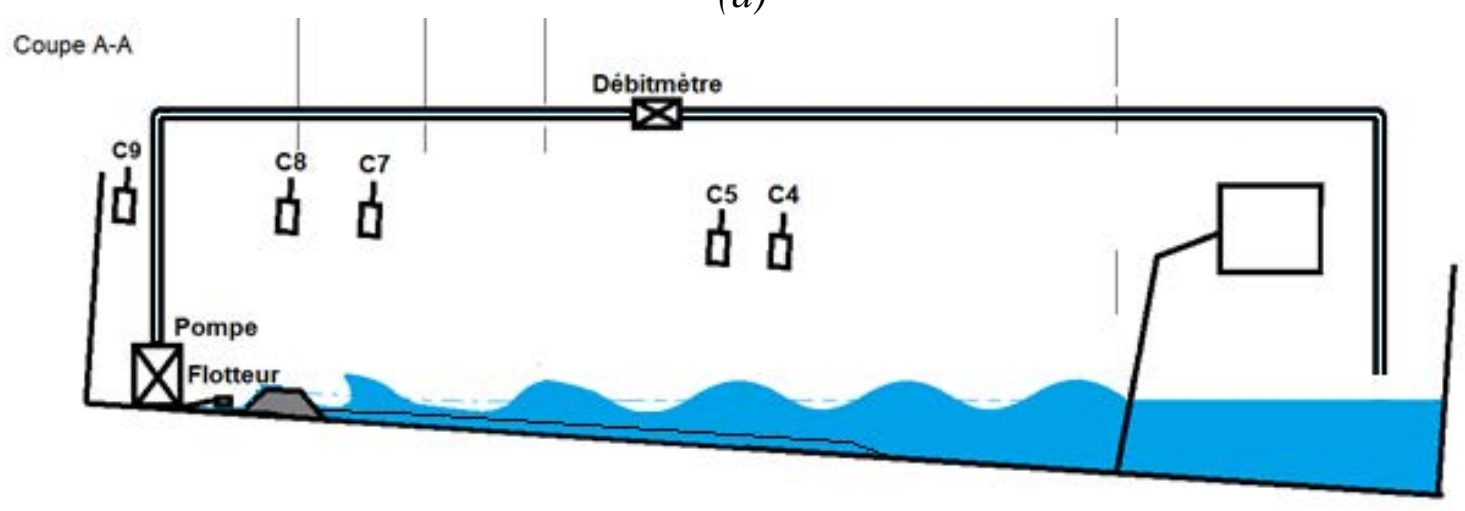

(b)

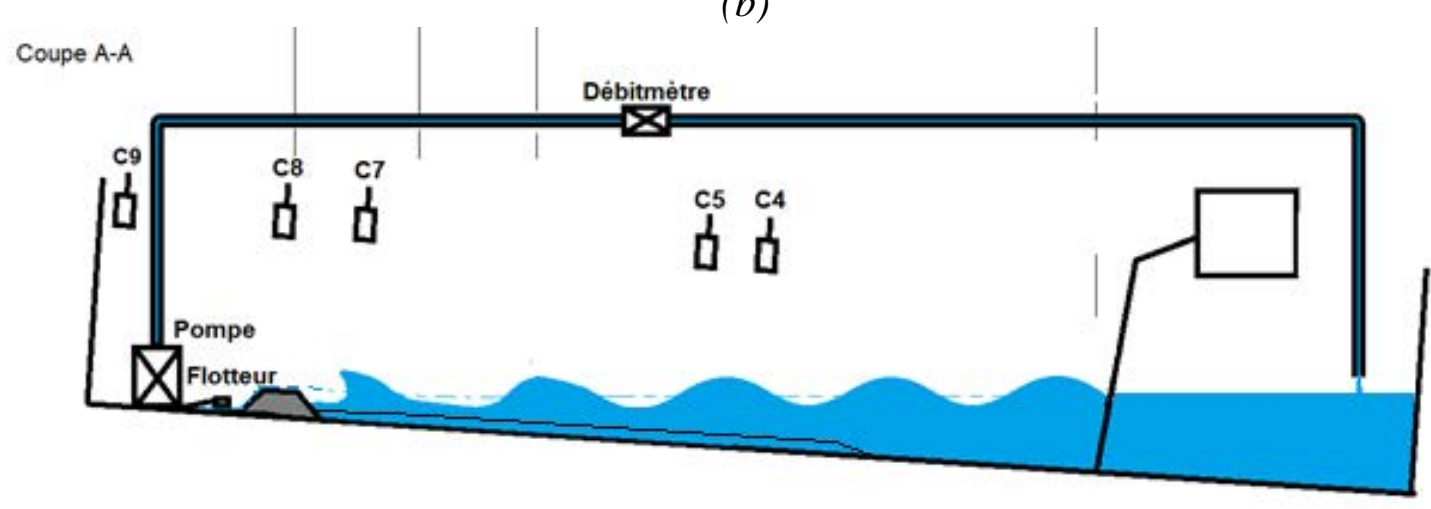

(c)

Figure 2. Dispositif expérimental pour : a) la surverse seule, b) le franchissement seul, c) les deux combinés.

Le schéma de ce dispositif est présenté sur la figure 2 pour chacune des 3 configurations. Les paramètres d'entrée à faire varier étaient la revanche $R c$ égale à la différence entre le niveau d'eau et la crête de digue et l'amplitude $H$ des vagues dans le cas du franchissement.

En sortie, il s'agissait alors de remonter au débit d'eau passé par-dessus la digue. 


\section{XVİ̀mes Journées Nationales Génie Côtier - Génie Civil \\ Le Havre 2020}

Dans le cas de la surverse seule (figure 2a), la pompe est active et nous obtenons une revanche négative de $2.3 \mathrm{~cm}$. L'écoulement est permanent et les paramètres d'entrée ne bougent pas.

Dans les cas de franchissement seul (figure 2b) et de franchissement/submersion (figure $2 \mathrm{c})$, la revanche $R c$ varie au cours du temps. Cela nous permet de pouvoir générer plusieurs jeux de résultats à partir d'une même acquisition. La revanche variant très lentement, les débits moyens (moyennés sur $10 \mathrm{~s}$, correspondant à 9 vagues pour notre fréquence $f=0.88 \mathrm{~Hz}$ ) mesurés correspondent à des variations de revanche de l'ordre de $5 \%$ au maximum.

Dans le cas de franchissement/submersion combiné (figure 2c), nous avons mesuré le débit en situation normale et en surélevant le fond pour le faire passer de 20 à $6 \mathrm{~cm}$ de manière à forcer le déferlement en amont de la digue et générer ainsi du set-up.

Les débits de surverse en présence de franchissement sont estimés à partir de la formule de HENDERSON (1966).

\section{Résultats}

\subsection{Reproduction du set-up sans obstacle}

La mesure de la surface libre a permis de montrer que la pente du canal n'avait pas d'impact sur l'amplification des vagues lors de la propagation (figure 3a). Par ailleurs, nous avons observé une légère différence entre nos mesures et les lois analytiques (figure $3 b)$ pour la gamme de profondeur $(2-6 \mathrm{~cm})$ que nous avons attribué à la non linéarité des vagues lors de la propagation. En diminuant l'amplitude des vagues $H_{0}$, nous avons bien vérifié cette supposition.

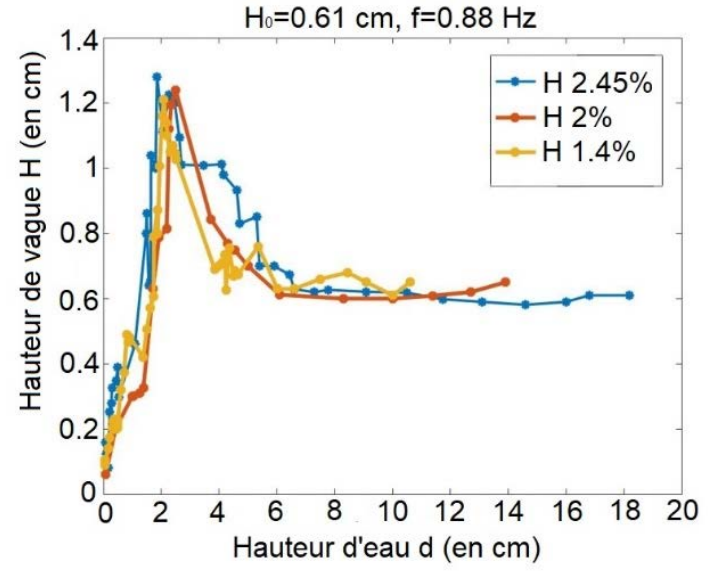

(a)

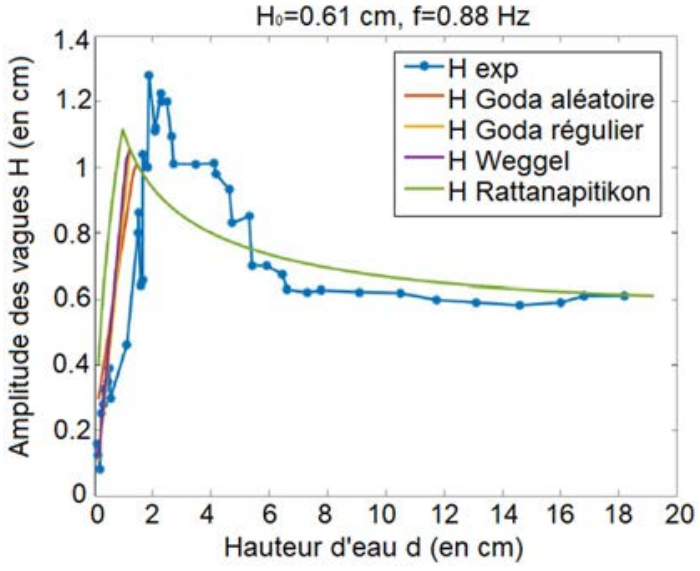

(b)

Figure 3. Amplification des vagues lors de la propagation : a) pour différentes pentes : $2.45 \%, 2 \%$ et $1.4 \%$; b) comparaison avec les formules analytiques (Goda aléatoire, Goda régulier, Waggel, Rattanapitikon). 


\section{Thème 7 - Risques côtiers}

En termes de set-up, nous retrouvons également un bon accord entre nos mesures et ce que prédisent les lois analytiques (figure 4). Pour le calcul du set-up, nous avons représenté la hauteur moyenne de l'eau sur la durée totale $N$ de l'acquisition ou sur 15 périodes de vague, après que le déferlement ne se soit produit.

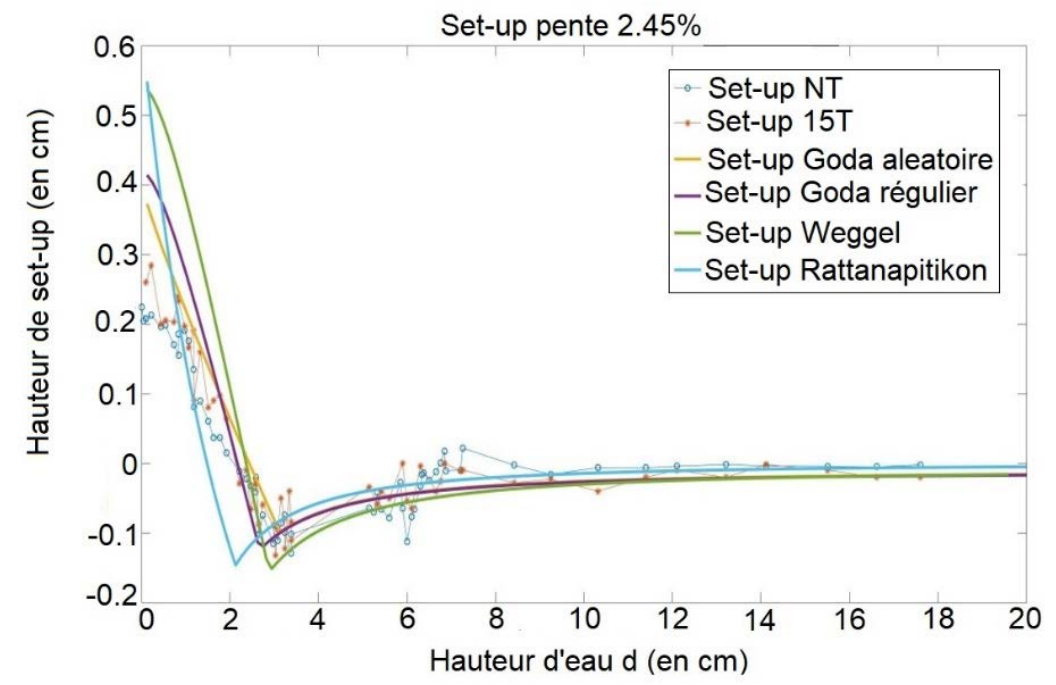

Figure 4. Set-up induit par les vagues en fonction de la profondeur d'eau locale

$$
\left(H_{0}=0.61 \mathrm{~cm} \text { et } f=0.88 \mathrm{~Hz}\right) \text {. }
$$

3.2 Surverse et/ou franchissement d'une digue à talus avec ou sans set-up

Nous avons dans un premier temps testé la surverse seule dans le canal (figure 2a). La pompe utilisée étant à débit imposé, nous avons complété nos mesures par un remplissage « jet d'eau» du canal. Les points de mesure sont superposés avec ce que prévoit la formule analytique de Henderson sur la figure 5.

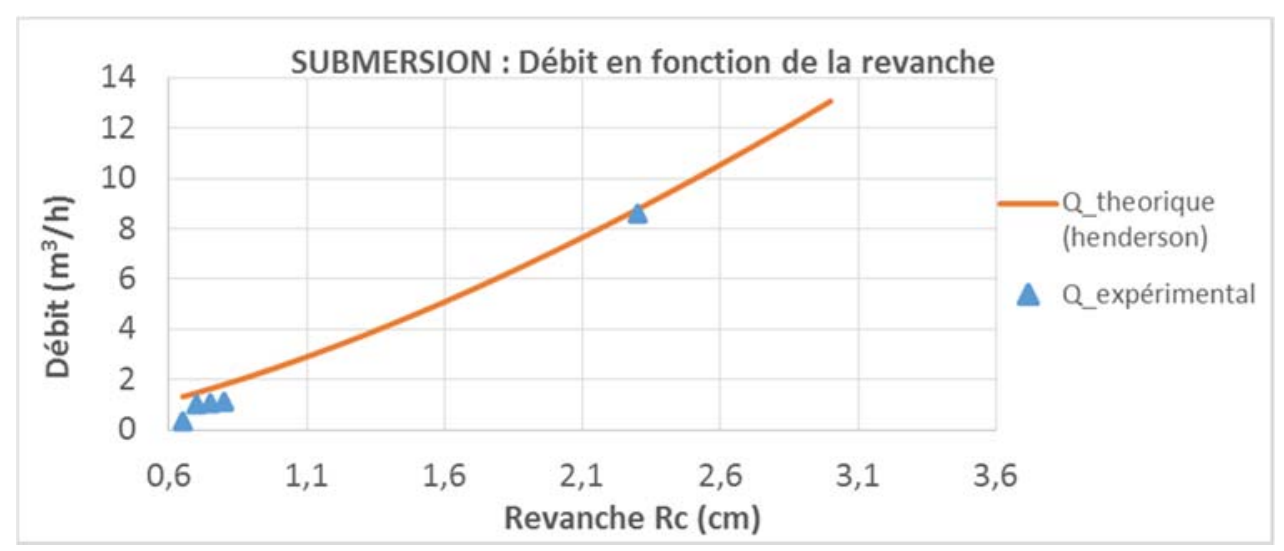

Figure 5. Débit de surverse en fonction de la revanche. 


\section{XVİ̀mes Journées Nationales Génie Côtier - Génie Civil \\ Le Havre 2020}

En essai de franchissement seul, nous avons pu faire varier l'amplitude des vagues et la revanche (toujours positive). Les résultats (figure 6) présentent un bon accord avec la formule empirique de Van Der Meer.

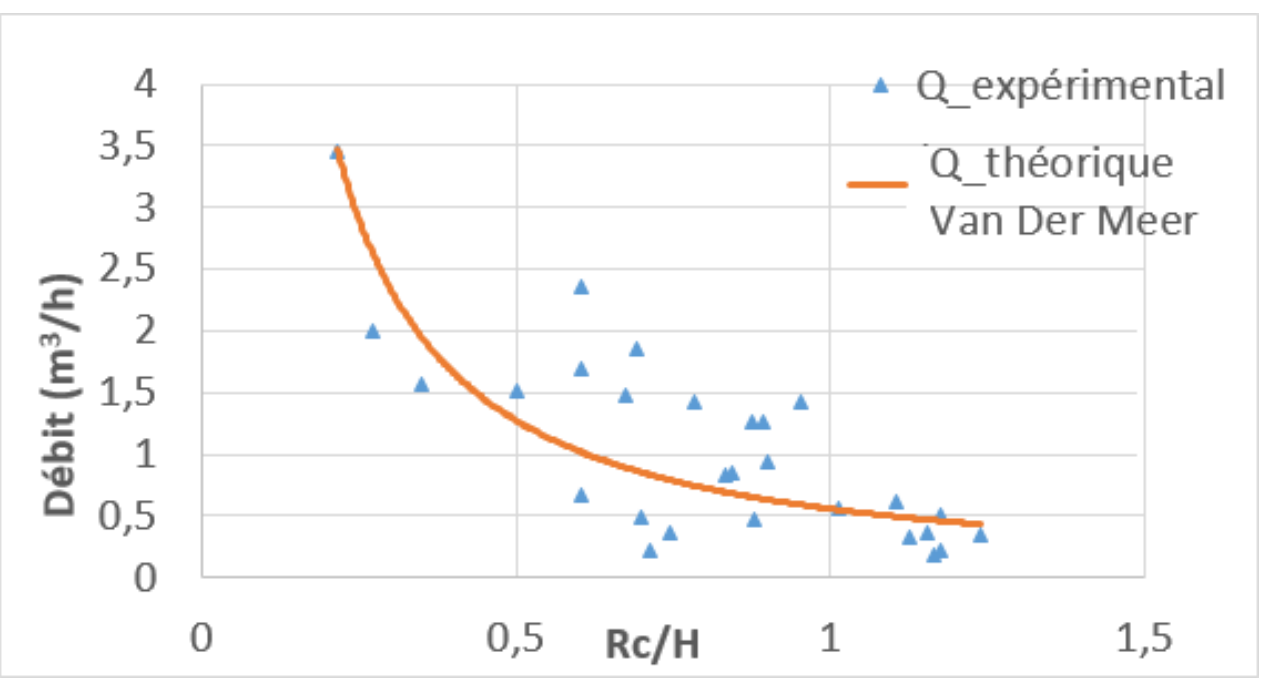

Figure 6. Débit de franchissement en fonction de Rc/H.

Enfin dans le cas de franchissement en présence de surverse, les résultats restent également assez proches de ce qui était attendu (figure 7).

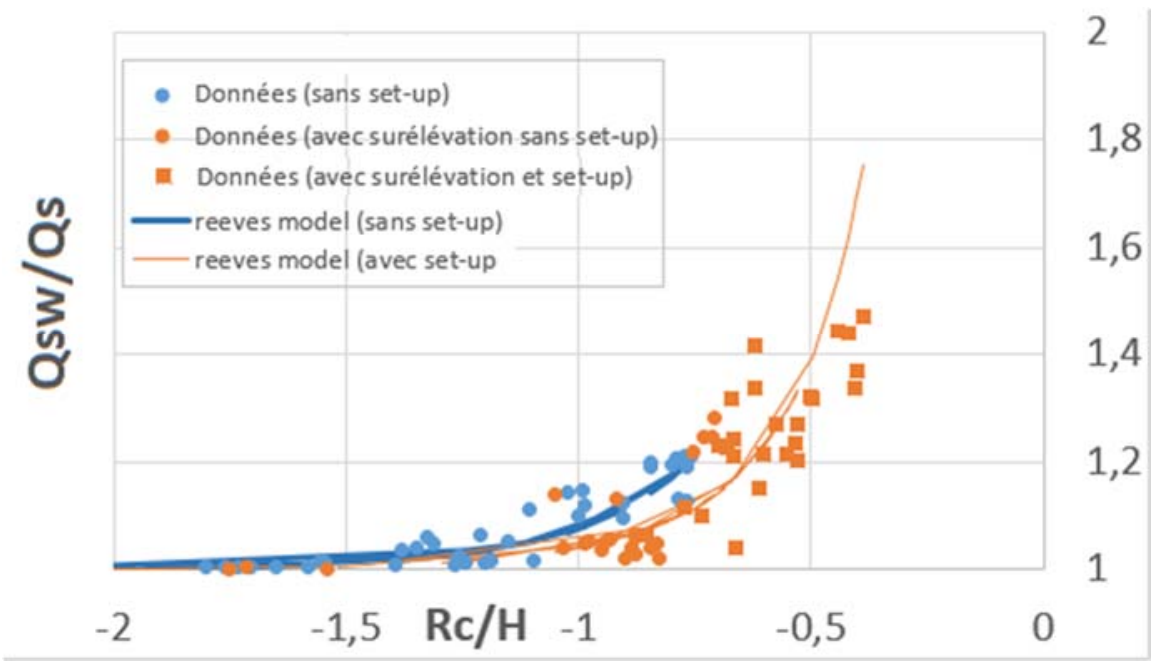

Figure 7. Rapport du débit total Qsw sur le débit de surverse Qs en fonction de Rc/H.

Nous retrouvons bien les résultats prédits par la formule empirique de Reeves (REEVES et al., 2008 ; HUGHES \& NADAL, 2009) à savoir que lorsque la revanche est faible et l'amplitude des vagues importante, le débit de franchissement devient non négligeable par rapport au débit de surverse. Dans notre cas, nous avons une contribution maximum du débit de franchissement qui atteint $30 \%$ du débit total. 


\section{Thème 7 - Risques côtiers}

Nous attribuons le décalage existant entre les 2 courbes (figure 7) à la zone de mesure de l'amplitude des vagues et de la revanche en pied de digue. En effet, nos mesures sont réalisées dans une zone où l'amplitude des vagues est maximum, proche d'un set-up minimum, ce qui a tendance à réduire le rapport $R c / H$ par rapport à la configuration sans set-up.

Afin de vérifier ces effets de variabilité spatiale du niveau d'eau en amont de la digue, nous avons réalisé des mesures pour déterminer la courbe de set-up correspondant au cas avec surélévation (figure 8).

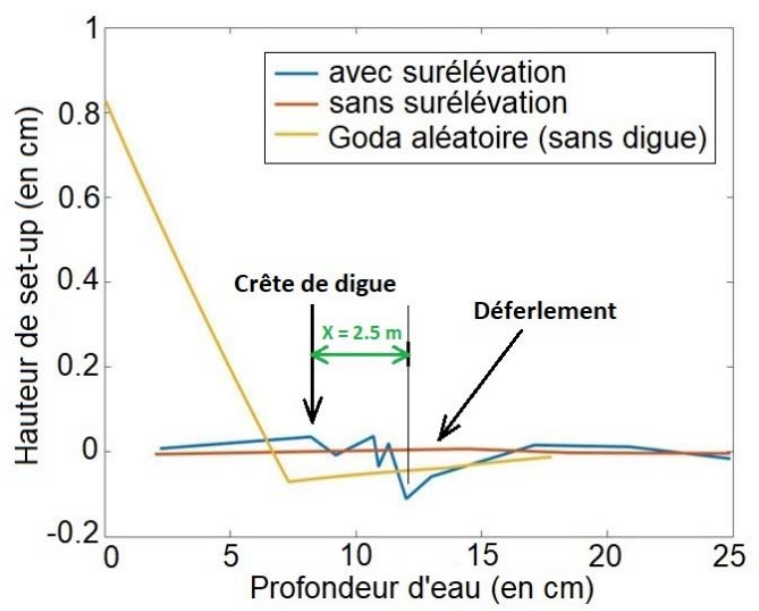

Figure 8. Courbe de set-up avec surélévation pour provoquer le déferlement.

Le set-down maximum, correspondant à la zone du déferlement, est positionnée à environ $2.5 \mathrm{~m}$ de la crête de digue ce qui correspond à une profondeur d'eau de $12 \mathrm{~cm}$. En utilisant la formule de Goda aléatoire, on constate que cette distance aurait pu permettre au set-up d'augmenter le niveau d'eau de 0.38 cm s'il n'y avait pas eu de surverse.

\section{Conclusion}

Le set-up a pu être reproduit en canal. La mesure des vagues a permis de montrer le caractère fortement non linéaire de la houle dans la zone du déferlement.

Le franchissement et la surverse ont été reproduits en canal et correspondent bien à ce qui était attendu.

Le franchissement en présence de surverse a bien montré que pour des gammes de $R c / H$ inférieures à 1 , le débit de franchissement doit être pris en compte et intégrer au débit total. Dans notre cas, nous obtenons une contribution maximale du débit de franchissement de l'ordre de 30\%. Cette valeur aurait pu être encore augmentée si nous avions pu travailler avec une revanche se rapprochant de zéro.

En cas de déferlement, le set-up apparaît mais n'a pas le temps de se développer puisqu'il est compensé par l'effet de surverse. La position du déferlement par rapport à la crête de 


\section{XVIèmes Journées Nationales Génie Côtier - Génie Civil \\ Le Havre 2020}

digue semble donc jouer un rôle très important dans le processus de franchissement/submersion. Il devra être étudié plus finement dans nos prochaines études.

\section{Références bibliographiques}

GODA Y. (1970). Numerical experiments on wave statistics with spectral simulation. Report of the Port and Harbour Research Institute, vol. 9, no. 3, pp 1-57.

HENDERSON F. M. (1966). Open channel flow. MacMillian Publishing Co., New York. HUGHES S. A., NADAL N. C. (2009). Laboratory study of combined overtopping and storm surge overflow of a levee. Coastal Engineering, Vol. 56, pp 244-259. https://doi.org/10.1016/j.coastaleng.2008.09.005

RATTANAPITIKON W., TOMOYA S. (2000). Verification and modification of Breaker Height Formulas. Coastal Engineering Journal, Volume 15, pp 389-406. https://doi.org/10.1142/S0578563400000195

REEVES D. E., SOLIMAN A., LIN P. Z. (2008). Numerical study of combined overflow and wave overtopping over a smooth impermeable seawall. Coastal Engineering, Vol. 55, pp 155-156. https://doi.org/10.1016/j.coastalgeng.2007.09.008

VAN DER MEER J. W., JANSSEN W. (1995). Wave run-up and wave overtopping at dikes. Kabayashi, Demirbilek (Eds), Wave Forces on Inclined and vertical Wall Structures. American Society of Civil Engineers, pp 1-27.

WEGGEL R. J. (1972). Maximum breaker height. Journal of Waterways, Harbour \& Coastal Eng. Div., Vol. 98, pp 529-547. https://doi.org/10.1061/9780872620490.024 
Thème 7 - Risques côtiers 